The subject of increasing attention for challenging new instrumentation requirements are resistive plate chambers - gas particle detectors operated, in their initial design, in limited streamer mode, under an uniform electric field. This schematic of a double gap RPC module has two chambers mounted facing each other in the same mechanical structure with independent high voltage and sharing a common readout plane of strips.

\section{Resistive plate chambers}

W ith present and future experiments in particle physics and astrophysics demanding detectors capable of good simultaneous space and time resolution over large areas, resistive plate chambers (RPCs) are prominent among generic detector designs. Interest and progress were reflected at the III International Workshop on Resistive Plate Chambers and Related Detector (RPC95) in Pavia (Italy) last fall.

RPCs are gas particle detectors operated, in their initial design, in limited streamer mode, under an uniform electric field. The accompanying figure shows a schematic of a double gap RPC module, consisting of two chambers facing each other, mounted in the same mechanical structure with independent high voltage and sharing a common readout plane of strips.

Each chamber is composed of two plates of bakelite, a phenolic polymer with high volume resistivity, separated by a $2 \mathrm{~mm}$ gap. The outer faces of the bakelite plates are coated with a conductive graphite paint on which the high voltage is applied. Planarity of the plates is assured by PVC spacers.

These detectors have been initially used as veto counters for cosmic radiation in non-accelerator (NADIR) and accelerator experiments (FENICE) and later exploited as muon detectors in large area cosmic ray physics (COVER PLASTEX) as well as in fixed target beauty production experiments - E771 at Fermilab and WA92 at CERN.

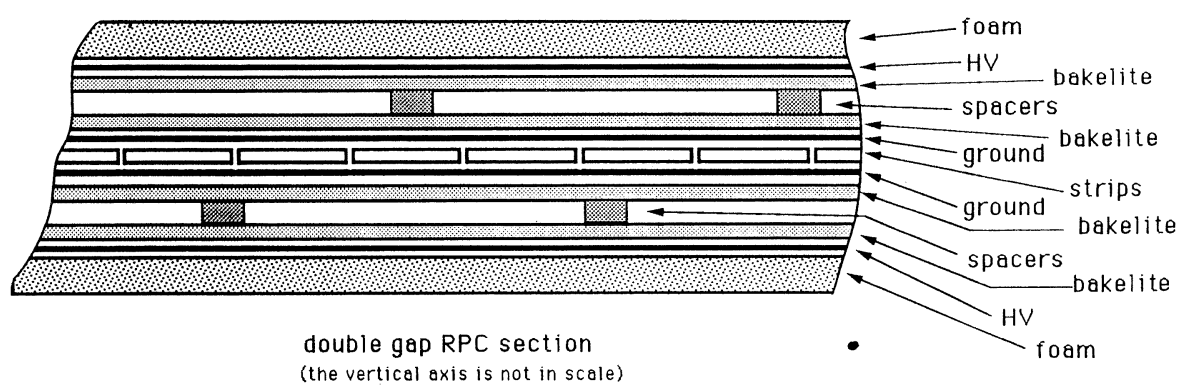

The L3 experiment at CERN's LEP electron-positron collider has used some $300 \mathrm{~m}^{2}$ of RPCs as muon trigger. At present RPCs are the approved muon detector baselines for ATLAS and CMS at CERN's LHC proton collider and $\mathrm{BaBar}$ at the SLAC B factory.

The increasingly severe environments in which the RPCs are supposed to operate - high intensities, large backgrounds, tight safety requirements,.... - require special attention.

The recent Workshop played an important role in bringing together experts and comparing different experimental approaches on major issues such as working mode (the merits of avalanche vs streamer mode), new electronics for avalanche mode, new freon gases and new resistive materials, wide gap and glass RPCs.

The Workshop was opened by $\mathrm{R}$. Santonico (developer of the first detector) who presented results on $2 \mathrm{~mm}$ gas gap bakelite RPCs operated with a new ecological freon $\left(\mathrm{C}_{2} \mathrm{H}_{2} \mathrm{~F}_{4}\right.$ instead of the standard $\mathrm{CF}_{3} \mathrm{Br}$ ) in a low gain gas condition. $\mathrm{He}$ discussed operating the RPC in the "avalanche mode", the precursor of the streamer. In this mode the signals are very small and front-end preamplification is needed: on the other hand the operating voltage can be kept below 10kV (where the streamer condition begins to appear) allowing a significant decrease of power dissipation. The collected saturation charge is of the order of 1 $\mathrm{pC}$ and typical time distributions have a jitter width of $4.5 \mathrm{~ns}$.

The rate capability has already been tested by the ATLAS collaboration (full efficiency up to $1-2 \mathrm{kHz} / \mathrm{cm}^{2}$ ) with the standard freon but it is hoped that such behaviour can be well reproduced with the new gas.

Interesting results were shown on the behaviour of different gap sizes $(2,5,6$ and $8 \mathrm{~mm})$ with melamine electrodes. The measurements, made on small RPCs, show that wide-gap RPCs have smaller dynamic range, leading to small average charge, high rate capability and low power consumption. However the operational voltage is high (16 to $19 \mathrm{kV}$, depending upon the gas mixture used).

The issue of surface treatment of the standard bakelite RPCs was discussed. Internal electrode treatment helps operation with lower current (a factor 4) and noise counting rate (a factor 10) compared to non-treated bakelite electrodes. 
Several talks then reported on the use of electrodes made of different material such as plastic (ABS) or glass.

The general feeling emerging from the Workshop was that much effort has gone into understanding the performances of the detector. Ongoing studies are likely to reveal RPC potential and versatility. First order problems have been solved but more systematic studies are needed. There was a consensus that a "new generation" of RPCs (new freons, new front-end electronics) will be able to operate with full efficiency at the high fluxes foreseen at CERN's LHC.

The Workshop was sponsored by the local Istituto Nazionale di Fisica Nucleare, the University of Pavia and the Fulbright Foundation.

From Sergio Ratti, Pavia

\section{QUANTUM MECHANICS Now you see it, now you don't}

$E_{\mathrm{o}}^{\mathrm{v}}$ ver since the development of quantum mechanics in the 1920 s as the ultimate description of microscopic objects, great minds have agonized over baffling conundrums which appear to arise when quantum behaviour has to be reconciled with everyday experience. However physicists who eat and drink quantum mechanics dismiss these agonies as unnecessary intellectual masochism. These physicists point out that such conundrums occur because nonquantum mechanical concepts and/or prejudices are imposed on a quantum scenario.

With the behaviour of individual quanta difficult to monitor, quantum physics has often had to rely on imaginary 'gedanken' experiments organized by such intellects as Werner Heisenberg or Richard Feynman. Now a delicate new experiment by an MIT/Innsbruck team using an atomic interferometer underlines the validity of these classic thought experiments.

The classic example of a quantum 'conundrum' is a beam of electrons particles - passing through two closely separated slits. A screen on the far side of the slits shows the characteristic bands of electron diffraction. In some places on the screen there are many electrons, while in others there are less - the screen is 'dark'. For these dark bands, blocking one slit increases the number of electrons coming through from the other. The electrons clearly behave as waves.

But the electrons are particles, and as such any one electron has to go either through one slit or the other, but not both. How can this be reconciled with the wave-like behaviour?
The ultimate quantum experiment. Electrons from a source (1) passing through a pair of slits (2) form a pattern on a screen (3).

Introducing a light source (4) allows one to tell which of the two slits a particular electron has passed through.

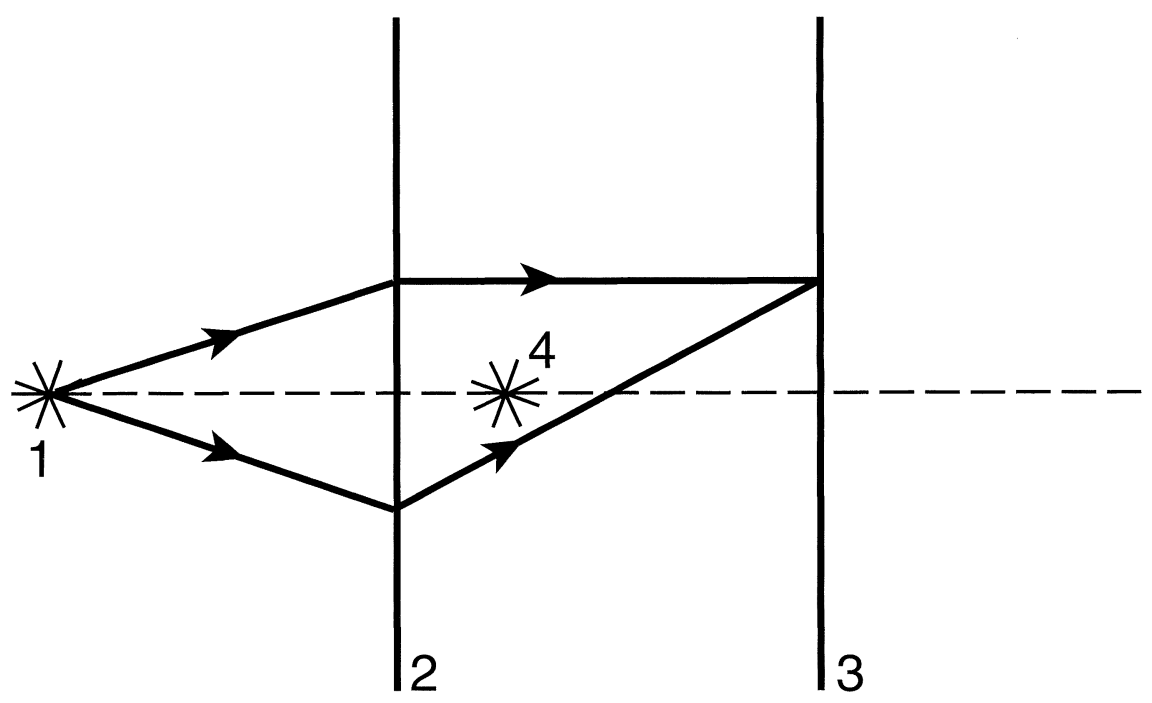

